\title{
Antidiabetic and antioxidant potentials of Euphorbia hirta leaves extract studied in streptozotocin-induced experimental diabetes in rats
}

\author{
Sorimuthu P. Subramanian, Subramanian Bhuvaneshwari and Gopalan S. Prasath \\ Department of Biochemistry, University of Madras, Guindy campus, Chennai 600 025, India
}

\begin{abstract}
Euphorbia hirta, commonly known as asthma weed, is a popular folk remedy for the treatment of various ailments. Recent studies have indicated that plant has potent antioxidant properties. As part of an ongoing programme to validate the use of some reputed herbs in Indian traditional medicines, the present study was aimed to evaluate the antidiabetic and antioxidant potentials of E. hirta leaves in streptozotocin-induced experimental diabetes in rats. Oral administration of $E$. hirta leaves extract ( $300 \mathrm{mg} / \mathrm{kg}$ b.w./rat/day) for a period of 30 days indicated the antidiabetic nature of the leaves extract. Determination of the lipid peroxides, hydroperoxides, and both enzymatic and nonenzymatic antioxidants evidenced the antioxidant potential of the leaves extract. Assay of enzymes such as serum aspartate transaminase (AST), serum alanine transaminase (ALT) and serum alkaline phosphatase (ALP) revealed the non-toxic nature of $E$. hirta leaves. The hypoglycemic activity of the leaves extract was comparable with gliclazide, a standard reference drug.
\end{abstract}

Key words: Antidiabetic - Antioxidant - Euphorbia hirta - STZ-induced diabetes - Gliclazide

Abbreviations: ALP, alkaline phosphatase; ALT, alanine transaminase; AST, aspartate transaminase; CAT, catalase; GPx, glutathione peroxidase; GST, glutathione-S-transferase; LSD, least significant difference; SOD, superoxide dismutase; STZ, streptozotocin.

\section{Introduction}

Diabetes mellitus is a complex multisystemic metabolic disorder as old as mankind that has reached epidemic proportions worldwide (Zimmet et al. 2001). It is characterized by persistent hyperglycemia as a result of relative or absolute lack of insulin or the actions of insulin. Hyperglycemia affects the metabolism of carbohydrates, protein and fat leading to structural changes in a wide range of cells subsequently leading to long-term complications and premature death. The prevalence of diabetes is strongly associated with a sedentary lifestyle and high calorie-nutrition and obesity (King et al. 1998). Currently available drugs for the treatment of diabetes such as sulfonylureas, biguanides, $\alpha$-glucosidase inhibitors, thiazolidenediones and insulin have a number of limitations such as adverse effects and high rates of secondary failure.

Correspondence to: Sorimuthu P. Subramanian, Department of Biochemistry, University of Madras, Guindy Campus, Chennai 600025 , India

E-mail: subbus2020@yahoo.co.in
The situation warrants the active search for the novel therapeutic agents preferably from natural sources.

Plants are omnipresent in the earth's environment and have been used for a large range of purposes including medicine, nutrition, flavoring, beverages, dyeing, repellants, fragrances, cosmetics, charms and various industrial uses (Smith and Winder 1996). India is one of the twelve megabiodiversity countries of the world having rich vegetation with a wide variety of plants with medicinal values. However, only few of them received scientific validation.

Euphorbia hirta is an herbaceous wild plant from the Euphorbiaceae family widely used in the Indian traditional medicine for various ailments. The plant is distributed in southern Western Ghats of India and Northern east coast of Tamil Nadu (Abdul Rahman et al. 2007). The plant has a worldwide distribution and its common names include cat's hair, asthma weed and milk weed. Its local name in the southern part of India is "Amman pacharisi". Whole plant or the leaf extract obtained from E. hirta is known to have multitude actions in the biological system. The plant is traditionally used as treatment of asthma and respiratory 
tract inflammation, cough, chronic bronchitis and other pulmonary disorders and proven pre-clinically for anti-inflammation, asthma, wound healing and diarrhea (Ogbulie et al. 2007).

The antidiabetic and free radical property of E. hirta flower extract has been studied in alloxan-induced diabetic mice (Kumar et al. 2010). However, literature survey revealed that no systematic studies have been carried out on antidiabetic effect of E. hirta leaves in experimental models. The aerial parts of the plants are reported to contain flavonoids such as quercetin, Euphorbianin and tannins (Euphorbins) (Aqil 1999). Several triterpenes, polyphenols and phytosterols have also been investigated (Sandeep Patil et al. 2009). Since the plant is reported to contain many biologically active ingredients with known pharmacological actions, the present study was conducted to explore the antidiabetic and antioxidant effects of oral administration of E. hirta leaves extract in STZ-induced experimental diabetic animal models.

\section{Materials and Methods}

\section{Plant material}

Fresh, mature E. hirta leaves were collected from a plant in Thiruvannamalai (Tamilnadu, India). Leaves were selectively removed using forceps. The plant was identified and authenticated by Dr. V. Kaviyarasan from Centre for Advanced studies in Botany, University of Madras. A voucher specimen has been deposited in the department herbarium.

\section{Preparation of plant extract}

The E. hirta leaves were shadow dried at room temperature and finely powdered in an electrical grinder and stored at $5^{\circ} \mathrm{C}$ until further use. The absence of flavonoids like quercetin and rutin in the aqueous extract is due to their non polar nature. Hence, the extraction was performed using less polar solvents (Loh et al. 2009). E. hirta leaves powder was delipidated with petroleum ether $\left(60-80^{\circ} \mathrm{C}\right)$ for overnight. It was then filtered, and filtrate was discarded and the residue was extracted with ethanol $(90 \% \mathrm{~V} / \mathrm{V})$ by soxhalation. Ethanol was evaporated in a rotary evaporator at $40-50^{\circ} \mathrm{C}$ under reduced pressure.

\section{Phytochemical screening}

Leaves extract was subjected to preliminary phytochemical screening for the presence of various plant constituents. The presence of alkaloids were inferred using Dragendroff's reagent; flavonoids by Shinoda's test; tannins with Ferric chloride test; and saponins by the production of stable foam and steroids with Libermann-Burchard reagent (Harborne 1998; Kokate 2005).

\section{Experimental animals}

Male albino Wistar rats weighing (160-180 g) were purchased from Tamilnadu Veterinary and Animal Sciences University (Chennai, India). The rats were housed in polypropylene cages lined with husk. It was renewed for every $24 \mathrm{~h}$. The rats were fed with commercial pelleted rats chow (Hindustan Lever Ltd., Bangalore, India), composition of $5 \%$ fat, $21 \%$ protein, $55 \%$ nitrogen-free extract, and $4 \%$ fiber $(\mathrm{w} / \mathrm{w})$ with adequate vitamin levels for the animals and had free access to water. The experimental rats were maintained in a controlled environment (12/12 h light/dark cycle and temperature $\left(30 \pm 2^{\circ} \mathrm{C}\right)$. The experiments were designed and conducted in strict accordance with the ethical norms approved by Ministry of Social justices and Environment, Government of India and Institutional Animal Ethical Committee guidelines (IAEC NO: 01/055/09). The rats were acclimatized for one week before starting the experiments.

\section{Experimental design}

Rats were fasted overnight and experimental diabetes was induced by intraperitoneal injection of streptozotocin (STZ) with a single dose of $50 \mathrm{mg} / \mathrm{kg}$ body weight (b.w.). STZ was dissolved in freshly prepared $0.1 \mathrm{M}$ cold citrate buffer, pH 4.5 (Rakieten et al. 1963). The control rats were injected with the citrate buffer. Because STZ is capable of inducing fatal hypoglycemia as a result of massive pancreatic insulin release, STZ-treated rats were allowed to drink $10 \%$ glucose solution overnight to overcome the drug-induced hypoglycemia (Palsamy and Subramanian 2008). Neither death nor any other adverse effect was observed. After 3 days for development and aggravation of diabetes, rats with moderate diabetes (i.e., blood glucose concentration above $250 \mathrm{mg} / \mathrm{dl}$ ) that exhibited glycosuria and hyperglycemia were selected for the experiment (Cánepa et al. 1990).

The animals were divided into four groups, comprising of six animals in each group as follows: control rats (group I), STZ-induced diabetic rats (group II), diabetic rats treated with $E$. hirta leaves extract ( $300 \mathrm{mg} / \mathrm{kg}$ b.w./rat/day) in aqueous solution orally for 30 days (group III), and diabetic rats given a single dose of gliclazide $(5 \mathrm{mg} / \mathrm{kg}$ b.w.) in aqueous solution orally for 30 days (group IV) (Pulido et al. 1997).

The change in body weight gain in all groups of rats was recorded at regular intervals. After 30 days of treatment, rats were deprived of food overnight and sacrificed by cervical dislocation. Blood was collected in sterilized tubes with and without anticoagulant. 


\section{Oral glucose tolerance test}

At the end of the experimental period, fasting blood samples were collected from all groups of rats. Four more blood samples were collected at 30,60,90 and 120 min intervals after the oral administration of glucose solution at a dosage of $2 \mathrm{~g} / \mathrm{kg}$ b.w. and the blood samples were collected with EDTA for the estimation of glucose.

\section{Biochemical parameters}

The biochemical parameters analyzed include blood glucose, urea, plasma insulin, protein, lipid peroxides, hydroperoxides, enzymatic and non enzymatic antioxidants. Serum creatinine and uric acid levels were also determined. The activities of aspartate transaminase (AST), alanine transaminase (ALT) and alkaline phosphatase (ALP) were assayed in the serum of control and experimental groups of animals. For the analyses of the above parameters commercially available kits and routine standardized procedures were adopted.

\section{Statistical analysis}

The results were expressed as mean \pm SD of six rats per group and statistical significance was evaluated by one-way ANOVA using SPSS (version 19.0) program followed by the post hoc test, least significant difference (LSD). Values were considered statistically significant when $p<0.05$.

\section{Results}

The preliminary phytochemical analysis of E. hirta leaves extract showed a positive response for the presence of alkaloids, flavonoids, glycosides, tannins, saponins and triterpenoids.

The effect of E. hirta leaves extract as well as gliclazide supplementation on glucose tolerance in control and experimental groups of rats receiving an oral glucose load is shown in Figure 1. The blood glucose concentration in the control rats was elevated to a maximum value at $60 \mathrm{~min}$ and declined to near basal levels at $120 \mathrm{~min}$, whereas, in STZinduced diabetic rats, the peak increase in blood glucose level was noticed even after $60 \mathrm{~min}$ and remained high over the next $60 \mathrm{~min}$. Oral treatment with $E$. hirta leaves extract as well as gliclazide to diabetic rats elicited significant $(p<0.05)$ decrease in blood glucose level at $60 \mathrm{~min}$ when compared with untreated diabetic rats.

Table 1 shows the effect of E. hirta leaves extract on the levels of biochemical and hematological parameters in the control and experimental groups of rats. The observed elevated levels of blood glucose, glycosylated hemoglobin, urea, uric acid and creatinine in the diabetic group of rats were reverted to near normal levels by the oral administration of E. hirta leaves extract. Conversely, the decreased levels of plasma insulin, hemoglobin and total protein in diabetic group of rats were elevated by the administration of E. hirta leaves extract to diabetic rats for 30 days. Urine

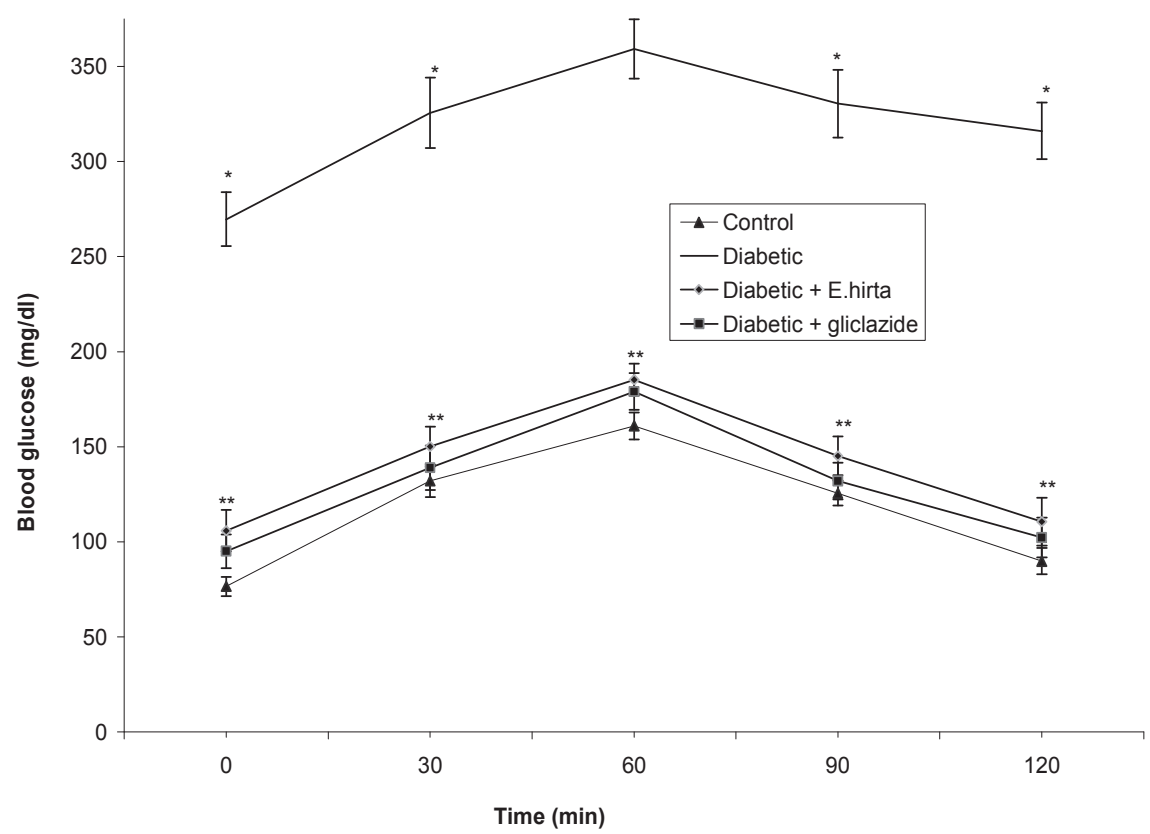

Figure 1. Effect of E. hirta leaves extract on the levels of blood glucose after an oral glucose load in the experimental groups of rats. Results are expressed as mean \pm S. D., $n=6$. One-way ANOVA followed by post hoc test LSD. $p<0.05$. The results were compared with * control, ${ }^{* *}$ diabetic control. 
Table 1. Effect of E. hirta leaves extract on the levels of biochemical and hematological parameters in the control and experimental groups of rats

\begin{tabular}{lcccc}
\hline Parameters & Control & Diabetic control & Diabetic + E. hirta & Diabetic + gliclazide \\
\hline Blood glucose $(\mathrm{mg} / \mathrm{dl})$ & $92.54 \pm 6.30$ & $301.51 \pm 39.17^{*}$ & $95.56 \pm 9.85^{* *}$ & $94.12 \pm 5.67^{* *}$ \\
Plasma insulin $(\mu \mathrm{U} / \mathrm{ml})$ & $12.53 \pm 0.92$ & $4.97 \pm 0.28^{*}$ & $9.04 \pm 0.16^{* *}$ & $10.42 \pm 0.82^{* *}$ \\
Hemoglobin $(\mathrm{g} / \mathrm{dl})$ & $13.01 \pm 4.17$ & $8.11 \pm 2.03^{*}$ & $11.29 \pm 2.97^{* *}$ & $11.86 \pm 2.54^{* *}$ \\
Glycosylated hemoglobin $(\% \mathrm{Hb})$ & $5.86 \pm 1.74$ & $13.11 \pm 4.05^{*}$ & $6.99 \pm 1.17^{* *}$ & $6.39 \pm 0.83^{* *}$ \\
Total protein $(\mathrm{g} / \mathrm{dl})$ & $8.23 \pm 0.94$ & $4.82 \pm 0.46^{*}$ & $7.87 \pm 0.43^{* *}$ & $8.01 \pm 0.64^{* *}$ \\
Blood urea $(\mathrm{mg} / \mathrm{dl})$ & $19.36 \pm 2.41$ & $36.43 \pm 2.87^{*}$ & $24.81 \pm 1.96^{* *}$ & $23.67 \pm 1.09^{* *}$ \\
Serum creatinine $(\mathrm{mg} / \mathrm{dl})$ & $0.69 \pm 0.07$ & $1.40 \pm 0.09^{*}$ & $0.79 \pm 0.04^{* *}$ & $0.63 \pm 0.03^{* *}$ \\
Serum uric acid $(\mathrm{mg} / \mathrm{dl})$ & $2.98 \pm 0.28$ & $7.53 \pm 0.47^{*}$ & $4.36 \pm 0.40^{* *}$ & $3.73 \pm 0.31^{* *}$ \\
Urine sugar & 0 & +++ & 0 & 0 \\
\hline
\end{tabular}

+++ indicates more than $2 \%$ sugar. Results are expressed as mean \pm S.D., $n=6$. One-way ANOVA followed by post hoc test LSD; $p<0.05$. The results were compared with ${ }^{\star}$ control; ${ }^{* *}$ diabetic control.

sugar which is observed in the diabetic group of rats was absent in leaves extract as well as gliclazide-treated diabetic group of rats. The results are comparable with gliclazide, an oral hypoglycemic drug.

Table 2 depicts the effect of E. hirta leaves extract on the levels of lipid peroxides and hydroperoxides in the plasma of control and experimental groups of rats. The levels of lipid peroxides and hydroperoxides are found to be elevated in plasma of diabetic group of rats. Oral administration of E. hirta leaves extract as well as gliclazide significantly decreased $(p<0.05)$ the levels of lipid peroxides and hydroperoxides to near normalcy.

Table 3 depicts the effect of E. hirta leaves extract on activities of enzymatic and non-enzymatic antioxidants in plasma of control and experimental groups of rats. Oral treatment with $E$. hirta leaves extract to STZ-induced diabetic rats resulted in significant increased $(p<0.05)$ activities of superoxide dismutase (SOD), catalase (CAT), glutathione peroxidase (GPx), and glutathione-S-transferase (GST) enzymes as well as showed a significant increase $(p<0.05)$ in levels of the non-enzymatic antioxidants.

Table 4 depicts the effect of E. hirta leaves extract on the activities of serum AST, ALT and ALP in control and experimental group of rats. Oral administration of E. hirta leaves extract significantly decreased $(p<0.05)$ the activities of these enzymes.

\section{Discussion}

STZ, an antibiotic produced by Streptomyces achromogenes, has been widely used for inducing diabetes in the experimental animals through its cytotoxic effects on pancreatic $\beta$ cells (Rakieten 1963). The mechanism behind is that STZ is preferentially uptaken by pancreatic beta cell via GLUT2 transporter and causes DNA alkylation followed by the activation of poly ADP ribosylation leading to depletion of cytosolic concentration of $\mathrm{NAD}^{+}$and ATP. Enhanced ATP dephosphorylation after STZ treatment supplies substrate for xanthine oxidase resulting in the formation of superoxide radicals and also NO moiety is liberated from STZ leading to the destruction of $\beta$ cells by necrosis (Szkudelski 2001).

Gliclazide is a second generation sulphonylurea derivative and is used as a reference drug to compare the efficacy of oral hypoglycemic drugs (Pulido et al. 1997). The acute toxicity studies indicated the non-toxic nature of E. hirta leaves extract.

The drug showed optimum activity at $300 \mathrm{mg} / \mathrm{kg}$ b.w and further increase in extract dose did not result in a further significant decrease in blood glucose levels, thus it appears that unlike insulin and other oral hypoglycemic drugs overdose of the drug may not cause hypoglycemia (data not shown).

The oral glucose tolerance test (OGTT) measures the body's ability to utilize glucose, the body's main source of energy. The

Table 2. Effect of E. hirta leaves extract on the levels of lipid peroxides and hydroperoxides in the plasma of control and experimental groups of rats

\begin{tabular}{lcccc}
\hline Parameters & Control & Diabetic control & Diabetic + E. hirta & Diabetic + gliclazide \\
\hline Lipid peroxides $(\mathrm{nmol} / \mathrm{ml})$ & $3.42 \pm 0.83$ & $7.23 \pm 0.87^{*}$ & $4.16 \pm 0.68^{* *}$ & $3.94 \pm 0.31^{* *}$ \\
Hydroperoxide $\left(10^{-5} \mathrm{mmol} / \mathrm{dl}\right)$ & $7.61 \pm 1.62$ & $12.93 \pm 1.84^{*}$ & $9.33 \pm 1.67^{* *}$ & $8.95 \pm 1.58^{* *}$ \\
\hline
\end{tabular}

Results are expressed as mean \pm S.D., $n=6$. One-way ANOVA followed by post hoc test LSD; $p<0.05$. The results were compared with * control; ${ }^{* *}$ diabetic control. 
Table 3. Effect of $E$. hirta leaves extract on activities of enzymatic and non-enzymatic antioxidants in plasma of control and experimental groups of rats

\begin{tabular}{|c|c|c|c|c|}
\hline Parameters & Control & Diabetic control & Diabetic + E. hirta & Diabetic + gliclazide \\
\hline SOD & $12.45 \pm 1.41$ & $5.73 \pm 0.74^{*}$ & $9.79 \pm 1.41^{* *}$ & $8.54 \pm 1.18^{* *}$ \\
\hline CAT & $79.27 \pm 4.17$ & $34.23 \pm 2.84^{*}$ & $72.21 \pm 4.39^{* *}$ & $70.93 \pm 4.82^{* \star}$ \\
\hline GPx & $8.87 \pm 0.72$ & $4.93 \pm 1.03^{*}$ & $7.41 \pm 0.53^{* *}$ & $7.22 \pm 0.49^{* *}$ \\
\hline GST & $7.07 \pm 1.22$ & $3.64 \pm 0.85^{*}$ & $6.18 \pm 0.81^{* *}$ & $5.98 \pm 0.79^{* *}$ \\
\hline a-tocopherol (mg/dl) & $3.82 \pm 0.91$ & $1.79 \pm 0.23^{*}$ & $2.32 \pm 0.84^{* *}$ & $2.41 \pm 0.21^{\star *}$ \\
\hline Vitamin C (mg/dl) & $1.84 \pm 0.31$ & $0.58 \pm 0.12^{*}$ & $1.43 \pm 0.34^{* *}$ & $1.59 \pm 0.17^{* *}$ \\
\hline Ceruloplasmin (mg/dl) & $25.88 \pm 2.74$ & $15.84 \pm 1.14^{*}$ & $20.13 \pm 2.71^{* *}$ & $19.27 \pm 1.68^{* *}$ \\
\hline Reduced glutathione (mg/dl) & $28.19 \pm 4.82$ & $16.72 \pm 2.35^{*}$ & $23.93 \pm 4.11^{* *}$ & $24.88 \pm 4.64^{* *}$ \\
\hline
\end{tabular}

Enzyme activities are expressed as: $50 \%$ inhibition of epinephrine auto oxidation/min/mg protein for SOD; $\mu$ mol of hydrogen peroxide decomposed $/ \mathrm{min} / \mathrm{mg}$ protein for CAT; $\mu \mathrm{mol}$ of glutathione oxidized $/ \mathrm{min} / \mathrm{mg}$ protein for GPx; $\mathrm{nmol}$ of CDNB conjugate formed $/ \mathrm{min} / \mathrm{mg}$ protein for GST. Results are expressed as mean \pm S.D., $n=6$. One-way ANOVA followed by post hoc test LSD; $p<0.05$. The results were compared with ${ }^{*}$ control; ${ }^{* *}$ diabetic control.

impaired glucose tolerance observed in STZ-induced diabetic group of rats were altered to near normal by the treatment with E. hirta leaves extract which indicates the insulin stimulatory effect of $E$. hirta leaves extract from remnant $\beta$ cells.

Blood glucose is a biochemical index widely used for the diagnosis and monitoring the prognosis of diabetes mellitus. Deficiency of insulin causes hyperglycemic status as a result of excessive production of endogenous glucose by hepatic as well as extra hepatic tissues through gluconeogenic and glycogenolytic pathways and reduced utilization of glucose by various tissues, a classical state of diabetes mellitus (Soling and Kleineke 1976). In the present study, oral treatment with E. hirta leaves extract as well as gliclazide lowered the level of blood glucose and improved the insulin level in STZ-induced diabetic rats.

The possible mechanism by which E. hirta leaves extract brings about its antidiabetic action may be by potentiating the insulin effect through the stimulation of insulin release from remnant pancreatic $\beta$ cells or its release from the bound form. In the above context a number of other plants have also shown similar mechanism (Arulselvan et al. 2006; Mahadeva Rao and Subramanian 2009).

The reduced glucose levels suggested that E. hirta leaves extract might exert insulin like action on peripheral tissues by either promoting glucose uptake metabolism or by inhibiting hepatic gluconeogenesis (Gray et al. 2000).

The level of total hemoglobin is found to be decreased in the diabetic group of rats and this may be due to increased formation of glycosylated hemoglobin. Glycosylated hemoglobin is considered as a golden marker for accurate and reliable measurement of fasting glucose. Chronic hyperglycemia results in glycosylation in which excess glucose nonenzymatically reacts with hemoglobin to form glycosylated hemoglobin (Koenig et al. 1976). This condition favors reduction in the level of hemoglobin and concomitant elevation in glycosylated hemoglobin, which is directly proportional to supraphysiological glucose (Alyassin and Ibrahim 1981). The observed decrease in the levels of glycosylated hemoglobin in diabetic rats treated with E. hirta leaves extract may be due to its hypoglycemic activity.

Urea is the chief end product of protein catabolism in the body. During the diabetic conditions, the catabolism of both hepatic as well as plasma proteins leads to the excessive accumulation of urea nitrogen in the blood. Moreover, the nitrogen homeostasis is also altered by elevated peripheral release of nitrogenous substances as well as elevated hepatic elimination of urea nitrogen. This results in the negative nitrogen balance. Oral administration of E. hirta leaves ex-

Table 4. Effect of E. hirta leaves extract on the activities of serum AST, ALT and ALP in control and experimental group of rats

\begin{tabular}{lcccc}
\hline Biochemical markers & Control & Diabetic control & Diabetic + E. hirta & Diabetic+ gliclazide \\
\hline Serum AST & $76.61 \pm 2.45$ & $133.50 \pm 2.54^{*}$ & $81.13 \pm 1.80^{* *}$ & $78.42 \pm 3.38^{* *}$ \\
Serum ALT & $16.22 \pm 0.73$ & $35.21 \pm 1.74^{*}$ & $15.23 \pm 0.55^{* *}$ & $14.42 \pm 0.91^{* *}$ \\
Serum ALP & $68.93 \pm 1.11$ & $107.79 \pm 3.02^{*}$ & $69.29 \pm 1.93^{* *}$ & $71.79 \pm 2.50^{* *}$ \\
\hline
\end{tabular}

Enzyme activities are expressed as: AST and ALT - $\mu \mathrm{mol}$ of pyruvate liberated/h/mg of protein; ALP - $\mu$ moles of phenol liberated/min/ $\mathrm{mg}$ of protein. Results are expressed as mean \pm S.D., $n=6$. One-way ANOVA followed by post hoc test LSD; $p<0.05$. The results were compared with ${ }^{*}$ control; ${ }^{* *}$ diabetic control. 
tract as well as gliclazide to experimental diabetic group of rats significantly normalized the altered levels of blood urea suggesting the beneficial role of E. hirta leaves in protein metabolism.

During STZ-induced experimental diabetes, the level of purines is elevated due to accelerated muscle wasting. These accumulated purines are the main source for the production of uric acid by the activity of xanthine oxidase (Anwar and Meki 2003). Various studies evidenced that increased oxidative stress is closely related to diabetes and its vascular complications (Baynes 1991). The elevated levels of circulating uric acid level may be an indicator that the body is trying to protect itself from the deleterious effects of free radicals by increasing the products of endogenous antioxidants, such as uric acid. Uric acid prevents oxidative modification of endothelial enzymes and preserves the ability of endothelium to mediate vascular dilatation in the face of oxidative stress (Becker 1993). In the present study, the increased level of serum uric acid observed in diabetic rats was restored to near normal by the administration of $E$. hirta leaves extract as well as gliclazide indicating the free radical scavenging activity of E. hirta leaves.

The level of total proteins is found to decrease in diabetic group of rats. The deficiency of insulin leads to defective amino acid/protein metabolism, which may be a more important factor than hyperglycemia in the etiology of some diabetic complications (Rosenlund 1993). STZ-induced diabetes in rat model indicates several alterations of amino acid metabolism, which may be attributed to increased muscle proteolysis, reduced protein synthesis, an energy-dependent process in the liver, and stimulated hepatic gluconeogenesis utilizing gluconeogenic amino acids (Fando et al. 1985). This results in the decrease of total protein content in STZ-induced rats. Oral administration of E. hirta leaves extract to diabetic rats significantly inhibits proteolysis caused by insulin deficiency and improves total protein levels to near normalcy. This property of $E$. hirta leaves extract is comparable to that of gliclazide Sunil et al. (2010) have reported that total protein level was found to be increased in STZ-induced diabetic mice treated with leaf, stem and flower extracts of $E$. hirta.

Serum creatinine was found to be increased in diabetic group of rats. Serum creatinine concentration is not only used to assess the impairment of kidney function but also in clinical chemistry as an end point to detect treatment related toxic effects of substances on kidney tissues (Bräunlich et al. 1997). However, administration of E. hirta leaves extract to diabetic group of rats significantly normalized their levels indicating the renal protective nature that occurs due to hyperglycemia.

Diabetes is associated with significant oxidative stress and that oxidative damage to tissues may be a contributory factor to several diabetic complications. As there is a strong correlation between oxidative stress and incidence of diabe- tes, antioxidant supplementation gears up the detoxification machinery thereby playing a pivotal role in the treatment of diabetes mellitus (Osawa and Kato 2005). STZ is known to generate free radicals and these free radicals undergo dismutation to hydrogen peroxide. Subsequently, highly reactive hydrogen radicals are formed by the Fenton reaction. The action of reactive oxygen species (ROS) with a simultaneous massive increase in cytosolic calcium concentration causes rapid destruction of $\beta$ cells (Szkudelski 2001).

The levels of lipid peroxides and hydroperoxides are found to be elevated in plasma of diabetic group of rats. Hydroperoxides are potentially toxic molecules that are capable of demolishing enzymes and cell membranes (Wang et al. 1996). The elevation in the level of hydroperoxides may be due to declined activities of antioxidant enzymes, which is a favorable cause for abandoned production of free radicals and subsequent production of lipid hydroperoxides (Matkovics et al. 1997). Oral administration of E. hirta leaves extract as well as gliclazide significantly decreased the levels of lipid peroxides and hydroperoxides to near normalcy indicating the free radical scavenging action of E. hirta leaves. This antioxidant activity might be due to the presence of phytochemical constituents e.g. flavonoid in the E. hirta leaves which acts as a strong superoxide radical and singlet oxygen quenchers. These observations suggest that E. hirta leaves extract possess antioxidant potential.

The activities of enzymatic antioxidants and the levels of non-enzymatic antioxidants in plasma in diabetic group of rats were found to be decreased. Enzymatic antioxidants are involved in detoxification of free radicals and peroxides formed during oxidative stress, including diabetes. Enzymatic antioxidants such as SOD, CAT, GPx, and GST are crucial cellular components of antioxidant defense system in the body, thus playing a crucial role in the maintenance of a balanced redox status (Evan and Littlewood 1998). Diminished activities of enzymatic antioxidants in diabetic rats have been reported (Miyazaki et al. 2007). The results of the present study indicated that oral treatment with $E$. hirta leaves extract to STZ-induced diabetic rats resulted in increased activities of SOD, CAT, GPx, and GST enzymes. This may be attributed to the free radical scavenging and antidiabetic activities of the extract of E. hirta leaves.

Vitamins $\mathrm{C}$ and $\mathrm{E}$ and reduced glutathione are known to be non-enzymatic antioxidants that are found to be decreased in diabetic state because of their free-radical scavenging property (Garg and Bansal 2000). The declined levels of vitamins $\mathrm{C}$ and $\mathrm{E}$ and reduced glutathione were due to the increased production of free radicals (Fang et al. 2002). However, oral administration of $E$. hirta leaves extract to diabetic group of rats showed a significant increase in levels of these non-enzymatic antioxidants, thereby suggesting the free radical scavenging potential of $E$. hirta leaves extract which in turn may be responsible for its antioxidant property. 
Ohaeri (2001) found that liver was necrotized in STZinduced diabetic rats. Therefore the observed increase in the activities of AST, ALT and ALP in the serum of diabetic rats might be mainly due to the leakage of these enzymes from the liver cytosol into the blood stream. On the other hand, treatment of the diabetic rats with E. hirta leaves extract caused reduction in the activity of these enzymes in plasma when compared to the diabetic group and consequently alleviate the liver damage.

A number of phytochemical constituents have been detected in the E. hirta Linn; tannins, gallic acid, quercitin, phenols, alcohols, alkaloids (Adedapo et al. 2005). A variety of herbs and herbal extracts contain different phytochemicals with biological activity that can be of valuable therapeutic index. Phytochemicals such as saponins, terpenoids, flavonoids and tannins found to inhibit cancer cell proliferation, regulate inflammatory, immune response and protect against lipid peroxidation (Liu 2003). Rupasinghe et al. (2003) have reported that saponins possess hypocholesterolemic, antidiabetic, antitumor, antiviral, antioxidative, anticarcinogenic, immunostimulatory and hepatoprotective properties. Phenolic compounds have cholesterol lowering effect (Leontowicz et al. 2002); flavonoids increases the insulin secretion from the $\beta$ cells of pancreas and this may explain the antidiabetic potential of the plants (Hii and Howell 1985).

In conclusion, the observed antidiabetic and antioxidant properties of the E. hirta leaves might be due to the synergistic effect of biologically active phytochemicals present in the leaves extract.

\section{References}

Rahuman A. A., Gopalakrishnan G., Venkatesan P., Geetha K. (2007): Larvicidal activity of some Euphorbiaceae plant extracts against Aedes aegypti and Culex quinquefasciatus (Diptera: Culicidae). Parasitol. Res. 102, 839-836

Adedapo A. A., Abatan M. O., Idowu S. O., Olorunsogo O. O. (2005): Effects of chromatography fractions of Euphorbia hirta on the rat serum biochemistry. Afr. J. Biomed. Res. 8, 185-189

Alyassin D., Ibrahim K. (1981): A minor hemoglobin fraction and the level of fasting blood glucose. J. Fac. Med. Baghdad 23, 373-380

Anwar M. M., Meki A. R. (2003): Oxidative stress in streptozotocininduced diabetic rats: effects of garlic oil and melatonin. Comp. Biochem. Physiol. Mol. Integr. Physiol. 135, 539-547 doi:10.1016/S1095-6433(03)00114-4

Aqil M. (1999): Euphorbianin, a new glycoside from Euphorbia hirta Linn. Global J. Pure Appl. Sci. 5, 371-373

Arulselvan P., Senthilkumar G. P., Sathish Kumar D., Subramanian S. (2006): Anti-diabetic effect of Murraya koenigii leaves on streptozotocin induced diabetic rats. Pharmazie 61, 874-877
Baynes J. W. (1991): Role of oxidative stress in development of complications in diabetes. Diabetes 40, 405-412 doi:10.2337/diabetes.40.4.405

Becker B. F. (1993): Towards the physiological functions of uric acid. Free Radic. Biol. Med. 14, 615-631 doi:10.1016/0891-5849(93)90143-I

Bräunlich H., Marx F., Fleck C., Stein G. (1997): Kidney function in rats after 5/6 nephrectomy (5/6 NX); effort of treatment with vitamin E. Exp. Toxicol. Pathol. 49, 135-139

Cánepa E. T., Llambías E. B., Grinstein M. (1990): Studies on regulatory mechanisms of heme biosynthesis in hepatocytes from normal and experimental-diabetic rats. Role of insulin. Biochem. Cell. Biol. 68, 914-921 doi:10.1139/090-136

Evan G., Littlewood T. A. (1998): Matter of life and cell death. Science 28, 1317 doi:10.1126/science.281.5381.1317

Fando J. L., Jolin T., Salinas M., Dominguez F., Herrera E. (1985): The effect of streptozotocin diabetes on brain protein synthesis in the rat. Diabete Metab. 11, 92-97

Fang J. C., Kinlay S., Beltrame J., Hikiti H., Wainstein M., Behrendt D., Suh J., Frei B., Mudge G. H., Selwyn A. P., Ganz P. (2002): Effect of vitamins $\mathrm{C}$ and $\mathrm{E}$ on progression of transplant-associated arteriosclerosis: a randomised trial. Lancet 359, 1108-1113 doi:10.1016/S0140-6736(02)08154-0

Garg M. C., Bansal D. D. (2000): Protective antioxidant effect of vitamins $\mathrm{C}$ and $\mathrm{E}$ in streptozotocin induced diabetic rats. Indian J. Exp. Biol. 38, 101-104

Gray A. M., Abdel-Wahab Y. H., Flatt P. R. (2000): The traditional plant treatment, Sambucus nigra (elder), exhibits insulin-like and insulin-releasing actions in vitro. J Nutr. 130, 15-20

Harborne J. B. (1998): Methods of extraction and isolation. In: Phytochemical Methods. pp. 60-66, Chapman and Hall, London

Hii C. S., Howell S. L. (1985): Effects of flavonoids on insulin secretion and $45 \mathrm{Ca} 2+$ handling in rat islets of Langerhans. J. Endocrinol. 107, 1-8 doi:10.1677/joe.0.1070001

King H., Aubert R. E., Herman W. H. (1998): Global burden of diabetes, 1995-2025: prevalence, numerical estimates, and projections. Diabetes Care 21, 1414-1431 doi:10.2337/diacare.21.9.1414

Koenig R. J., Peterson C. M., Jones R. L., Saudek C., Lehrman M., Cerami A. (1976): Correlation of glucose regulation and hemoglobin AIc in diabetes mellitus. N. Engl. J. Med. 295, 417-420 doi:10.1056/NEJM197608192950804

Kokate C. K. (2005): Practical Pharmacognosy. pp. 107-111, Vallabh Prakashan, New Delhi, India

Kumar S., Malhotra R., Kumar D. (2010): Antidiabetic and free radicals scavenging potential of Euphorbia hirta flower extract Indian J. Pharm. Sci. 72, 533-537 doi:10.4103/0250-474X.73921

Leontowicz H., Gorinstein S., Lojek A., Leontowicz M., Ciz M., SolivaFortuny R., Park Y. S., Jung S. T., Trakhtenberg S., Martin-Belloso O. (2002): Comparative content of some bioactive compounds in apples, peaches and pears and their influence on lipids and antioxidant capacity in rats. J. Nutr. Biochem. 13, 603-610 doi:10.1016/S0955-2863(02)00206-1 
Liu R. H. (2003): Health benefits of fruit and vegetables are from additive and synergistic combinations of phytochemicals. Am. J. Clin. Nutr. 78, S517-520

Loh D. S., Er H. M., Chen Y. S. (2009): Mutagenic and antimutagenic activities of aqueous and methanol extracts of Euphorbia hirta. J. Ethnopharmacol. 126, 406-414 doi:10.1016/j.jep.2009.09.025

Mahadeva Rao U. S., Subramanian S. (2009): Biochemical evaluation of antihyperglycemic and antioxidative effects of Morinda citrifolia fruit extract studied in streptozotocin-induced diabetic rats. Med. Chem. Res. 18, 433-446 doi:10.1007/s00044-008-9140-1

Matkovics B., Kotorman M., Varga I. S., Hai D. Q., Varga C. (1997): Oxidative stress in experimental diabetes induced by streptozotocin. Acta Physiol. Hung. 85, 29-38

Miyazaki Y., Kawano H., Yoshida T., Miyamoto S., Hokamaki J., Nagayoshi Y., Yamabe H., Nakamura H., Yodoi J., Ogawa H. (2007): Pancreatic B-cell function is altered by oxidative stress induced by acute hyperglycaemia. Diabet. Med. 24, 154-160 doi:10.1111/j.1464-5491.2007.02058.x

Ogbulie J. N., Ogueke, C. C., Okoli I. C., Anyanwu B. N. (2007): Antibacterial activities and toxicological potentials of crude ethanolic extracts of Euphorbia hirta. Afr. J. Biotechnol. 6, $1544-1548$

Ohaeri O. C. (2001): Effect of garlic oil on the levels of various enzymes in the serum and tissue of streptozotocin diabetic rats. Biosci. Rep. 21, 19-24 doi:10.1023/A:1010425932561

Osawa T., Kato Y. (2005): Protective role of antioxidative food factors in oxidative stress caused by hyperglycemia. Ann. N. Y. Acad. Sci. 1043, 440-451 doi:10.1196/annals. 1333.050

Palsamy P., Subramanian S. (2008): Resveratrol, a natural phytoalexin, normalizes hyperglycemia in streptozotocin-nicotinamide induced experimental diabetic rats. Biomed. Pharmacother. 62, 598-605 doi:10.1016/j.biopha.2008.06.037

Pulido N., Suarez A., Casanova B., Romero R., Rodriguez E., Rovira A. (1997): Gliclazide treatment of streptozotocin diabetic rats restores GLUT4 protein content and basal glucose uptake in skeletal muscle. Metabolism 46, 10-13 doi:10.1016/S0026-0495(97)90310-3
Rakieten N., Rakieten M. L., Nadkarni M. R. (1963): Studies on the diabetogenic action of streptozotocin (NSC-37917). Cancer Chemother. Rep. 29, 91-98

Rosenlund B. L. (1993): Effects of insulin on free amino acids in plasma and the role of the amino acid metabolism in the etiology of diabetic microangiopathy. Biochem. Med. Metab. Biol. 49, 375-391

doi:10.1006/bmmb.1993.1038

Rupasinghe H. P., Jackson C. J., Poysa V., Di Berardo C., Bewley J. D., Jenkinson J. (2003): Soyasapogenol A and B distribution in soybean (Glycine max L. Merr.) in relation to seed physiology, genetic variability, and growing location. J. Agric. Food Chem. 51, 5888-5894 doi:10.1021/jf0343736

Sandeep Patil B., Nilofar Naikwade S., Chandrakant Magdum S. (2009): Review on phytochemistry and pharmacological aspects of Euphorbia hirta Linn. J. Pharma. Res. Health Care $1,113-133$

Smith R. J., Winder M. L. (1996): Medicinal garden. In: The National Herb Garden Guidebook. (Ed. R. Ober), pp. 61-71, The Herb Society of America Springfield, USA

Soling H. D., Kleineke J. (1976): Species dependent regulation of hepatic gluconeogenesis in higher animals. In: Gluconeogenesis: its Regulation in Mammalian Species. (Eds. R. W. Hanson and M. A. Mehlman), pp. 369-462, Wiley Interscience, New York

Sunil Kumar Rashmi, Kumar D. (2010): Evaluation of antidiabetic activity of Euphorbia hirta Linn. in streptozotocin induced diabetic mice. Indian J. Natural Products and Resources 2, 200-203

Szkudelski T. (2001): The mechanism of alloxan and streptozotocin action in B cells of the rat pancreas. Physiol. Res. 50, 537-546

Wang W., Pang C. C., Rogers M. S., Chang A. M. (1996): Lipid peroxidation in cord blood at birth. Am. J. Obstet. Gynecol. 174, 62-65 doi:10.1016/S0002-9378(96)70374-5

Zimmet P., Alberti K. G., Shaw J. (2001): Global and societal implications of the diabetes epidemic. Nature 414, 782-787 doi:10.1038/414782a

Received: September 24, 2010

Final version accepted: April 6, 2011 